\title{
'Outside-in' demyelination in MS
}

Early

Subarachnoid space

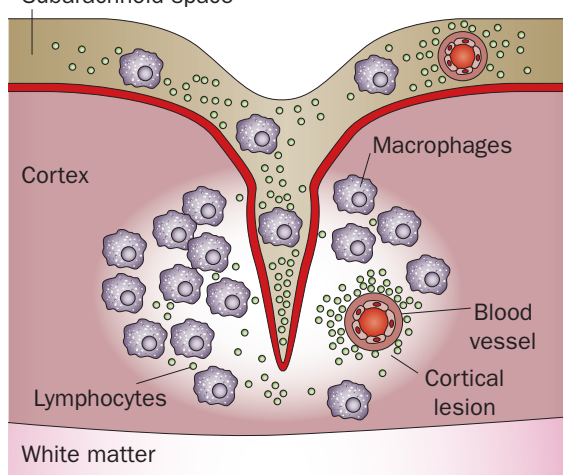

Chronic

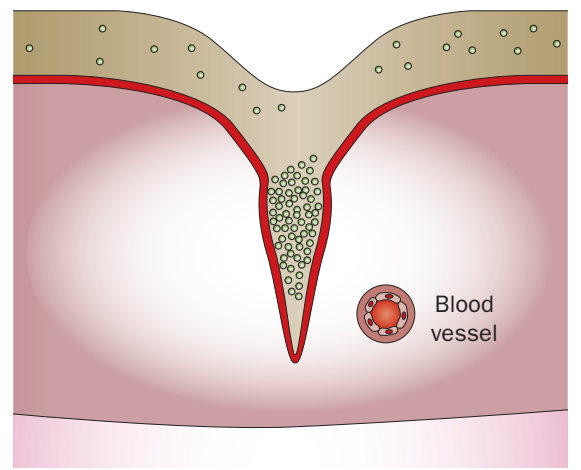

Schematic illustration showing levels of inflammation in patients with early-stage multiple sclerosis (left panel) and those with chronic disease (right panel). Adapted from an image provided by C. Lucchinetti.

The earliest stage of multiple sclerosis (MS) is characterized by inflammatory lesions in the cortex, according to the results of a recent study published in The New England Journal of Medicine. The findings support an 'outside-in' model of demyelination-beginning in the subarachnoid space and cortex then extending into the white matter - and are the first to suggest that MS is initiated by an inflammatory demyelinating event in the cortex.

MS was thought to be a demyelinating disease predominantly of the white matter. "Recent imaging data coupled with pathological studies confirm that MS is also a gray matter disease with extensive cortical involvement," says Claudia Lucchinetti, joint lead researcher of the study with Richard Ransohoff. "In fact, cortical damage is the strongest predictor of progressive disability in patients with MS. It is, therefore, critical to understand its pathology and pathogenesis."

Lucchinetti, Ransohoff and their colleagues sought to characterize cortical pathology in patients at the earliest stage of MS. They assessed biopsies taken from patients at their diagnostic evaluation and identified 138 samples that contained cortex, 53 of which had evidence of cortical demyelination. $81 \%$ of the biopsied patients who underwent follow-up examination were diagnosed with MS.
The researchers observed a wide spectrum of cortical lesions in the biopsy samples, similar to those seen in autopsy samples from patients with chronic MS. However, unlike the cortex in patients with chronic MS, cortex samples from patients with early MS had lymphocytic and macrophage infiltrates within the lesions. "Evidence of neurodegeneration occurred on a background of inflammation, which is consistent with MS being a primary inflammatory demyelinating disorder, rather than a primary neurodegenerative disorder," explains Lucchinetti.

The researchers are currently analyzing the nature of the cortical inflammatory infiltrates and comparing gray matter inflammation with that observed in the white matter. They hope that characterization of these early lesions may help to identify novel inflammatory targets for the treatment of MS. "Our work underscores the urgency in identifying improved imaging techniques that can 'see' the cortical lesions early in the disease process," says Lucchinetti. She asserts that imaging of cortical damage in patients with early MS will enable researchers to assess the efficacy of current and novel therapies to control cortical inflammation.

\section{Katy Malpass}

Original article Lucchinetti, C. F. et al. Inflammatory cortical demyelination in early multiple sclerosis. N. Eng. J. Med. 365, 2188-2197 (2011) 